\title{
Concomitant Retroperitoneal Intramuscular Hemangioma and Intravascular Papillary Endothelial Hyperplasia: An Unusual Case Report
}

\author{
Ching-Yueh Lin', Yu-Chun Lin², Liang-Cheng Chen'1, Yung-Tsan Wu ${ }^{1}$ \\ ${ }^{1}$ Department of Physical Medicine and Rehabilitation, Tri-Service General Hospital, National Defense Medical \\ Center, Taipei, Taiwan \\ ${ }^{2}$ Department of Pathology, Tri-Service General Hospital, National Defense Medical Center, Taipei, Taiwan \\ Email: linchingyueh@gmail.com
}

Received 14 April 2014; revised 2 May 2014; accepted 11 May 2014

Copyright $(2014$ by authors and Scientific Research Publishing Inc.

This work is licensed under the Creative Commons Attribution International License (CC BY).

http://creativecommons.org/licenses/by/4.0/

(c) (i) Open Access

\begin{abstract}
Primary retroperitoneal tumors are rare, and benign retroperitoneal tumors are exceptionally rare. Herein, we present a very rare case of benign concomitant retroperitoneal intramuscular hemangioma and intravascular papillary endothelial hyperplasia, with the unusual presentation of lumbosacral plexopathy. After surgical treatment and rehabilitation, the patient recovered uneventfully. This case report should serve to remind physicians of the rare condition of a retroperitoneal tumor leading to a neurological deficit.
\end{abstract}

\section{Keywords}

Hemangioma, Intravascular Papillary Endothelial Hyperplasia (IPEH), Lumbosacral Plexopathy, Masson's Hemangioma, Retroperitoneal Tumor

\section{Introduction}

Primary retroperitoneal tumors are rare, accounting for only $0.07 \%$ to $0.6 \%$ of all tumors at the same location, and $62 \%$ to $82 \%$ of these tumors have been reported to be malignant [1]. Benign retroperitoneal tumors are rare, and vascular malformations such as hemangioma are exceptionally rare comprising only $0.5 \%$ to $2 \%$ of all retroperitoneal tumors [2]. In addition, intravascular papillary endothelial hyperplasia (IPEH) is an exceptionally rare disease, with only one case arising from the retroperitoneum published in the literature [3]. Herein, we 
present an unusual case with concurrent intramuscular hemangioma and IPEH in the retroperitoneum complicated with right side lumbosacral plexopathy.

\section{Case Report}

A 56-year-old male with no systemic diseases or any history of surgery or trauma before this admission presented with abrupt pain in his right lower limb for 1 month, followed by right leg numbness 3 weeks later. A physical examination revealed a painful sensation with hypoesthesia in the right hip to lateral thigh, and a mild reduction in strength of the hip flexors and knee extensors. The X-ray and magnetic resonance imaging (MRI) of the lumbosacral spine showed no abnormalities, and the laboratory work-up was essentially normal. Lumbar strain was initially impressed, and he received conservative treatment for 4 weeks. However, he did not respond to this treatment, so advanced imaging was arranged.

MRI showed a large lobulated mass with heterogeneous intensity measuring $5 \times 5 \times 21 \mathrm{~cm}$ (anteroposterior diameter $\times$ width $\times$ height) along the right iliacus muscle. The mass was hypointense on T1-weighted images and hyperintense on fat saturation T2-weighted images, combined with several hypointense dots inside the mass (Figure 1). In addition, heterogeneous contrast enhancement of the mass on T1-weighted imaging was seen, and therefore a retroperitoneal tumor of vascular origin was highly suspected (Figure 2). To obtain pathological

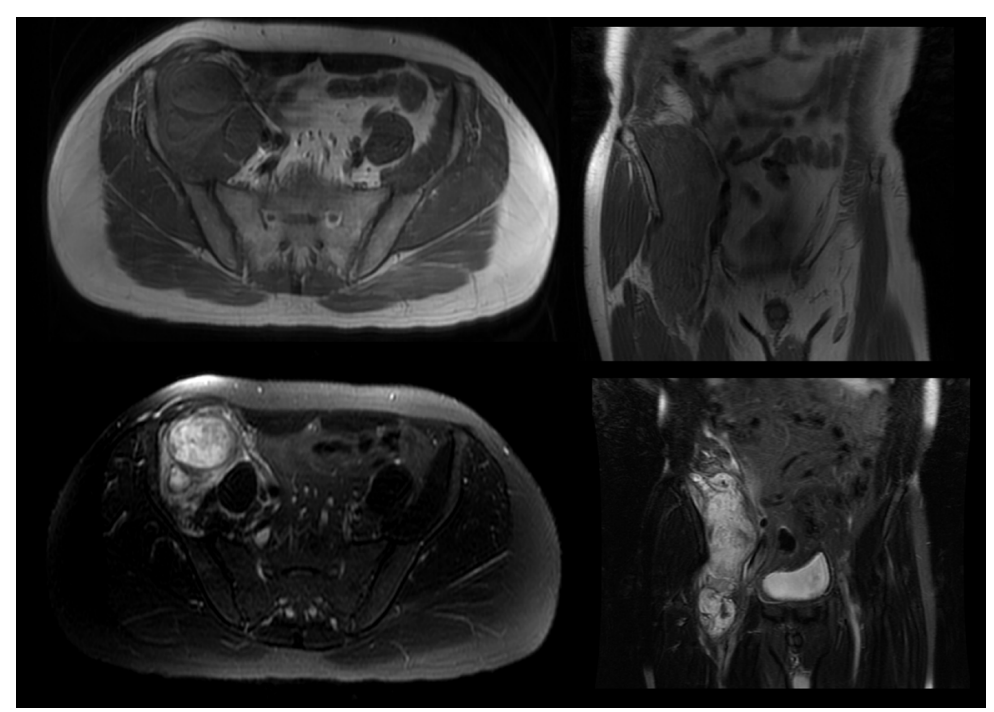

Figure 1. Magnetic resonance imaging of the pelvis. Top: A hypointense, lobulated mass $(5 \times 5 \times 21 \mathrm{~cm}$, anteroposterior diameter $\times$ width $\times$ height $)$ along the right iliacus muscle was shown on T1-weighted images (T1WI). Bottom: A heterogeneous, hyperintense mass with several hypointense dots inside and hypointense lining of the capsule were shown on fat saturation T2weighted images (FS-T2WI).
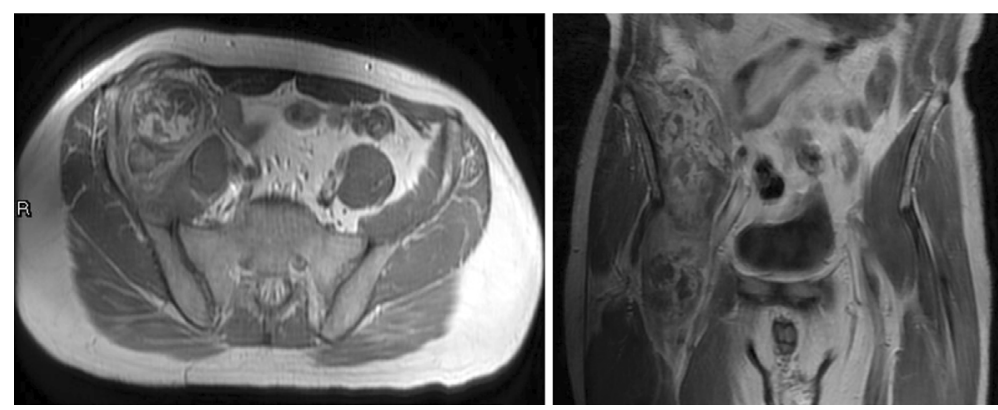

Figure 2. Post-gadolinium axial and coronal T1-weight images showed heterogeneous contrast enhancement of the mass, causing medial dislocation of the right iliac artery and vein. 
evidence and to prevent neurological deterioration, laparoscopic excision of the tumor was performed, and partial tumor invasion to the iliacus muscle was noted during the procedure.

Grossly, the tumor was a partially encapsulated cystic lesion containing friable thrombus-like material. Attached skeletal muscle tissue was also evident. Microscopically, an intramuscular hemangioma composed of one cystic dilated vascular channel and numerous associated irregular small vessels were seen. Thrombi formation with organization and papillary endothelial hyperplasia were also evident. Associated reactive myofibroblasts and capillary proliferation were seen in addition to the tumor (Figure 3). Based on the histopathological findings, an intramuscular hemangioma with IPEH was indicated.

The patient recovered uneventfully from the surgery except for persistent paresthesia in his right lateral thigh and muscle atrophy in his right vastusmedialis muscle. The muscle strength of his right quadriceps muscle was $3 / 5$, and electrophysiological studies indicated right lumbosacral plexopathy. After intensive rehabilitation for 3 months, the strength of his right quadriceps muscle improved from $3 / 5$ to $4 / 5$, and he could perform most daily activities independently. After 5 years of follow-up, the neurological sequela was minimal and no recurrence of the tumor was noted.

\section{Discussion}

To the best of our knowledge, this is the first reported case of concurrent retroperitoneal intramuscular hemangioma and IPEH-induced right lumbosacral plexopathy. Retroperitoneal hemangiomas are rare, and classified as cavernous, capillary, venous, and mixed types, with the last two types being extremely rare. They are usually asymptomatic except for tumor-related compression of surrounding tissue as the tumor grows. Although computed tomography and MRI are the standard methods for evaluation, the preoperative differentiation of malignant characteristics by imaging is often difficult. To prevent the tumor from rupturing/bleeding and to aid in differentiation, surgery is recommended [2].

IPEH, also known as Masson’s hemangioma, was first defined by Masson as a true neoplasm involving degenerative changes including thrombosis and necrosis, occurring when it expands beyond its blood supply [4]. In 1932, Henschen suggested the endothelial proliferation to be more like a reactive process, with endothelial proliferation and organization surrounding the thrombosis occurring during venous stasis [5]. In 1976, Clearkin and Enzinger proposed the term "IPEH", which was widely accepted thereafter [6]. To date, the exact pathogenesis of IPEH has not been clarified [7].

In a literature review [7]-[12], 23 cases of IPEH in the intraabdominal region were found, including the current
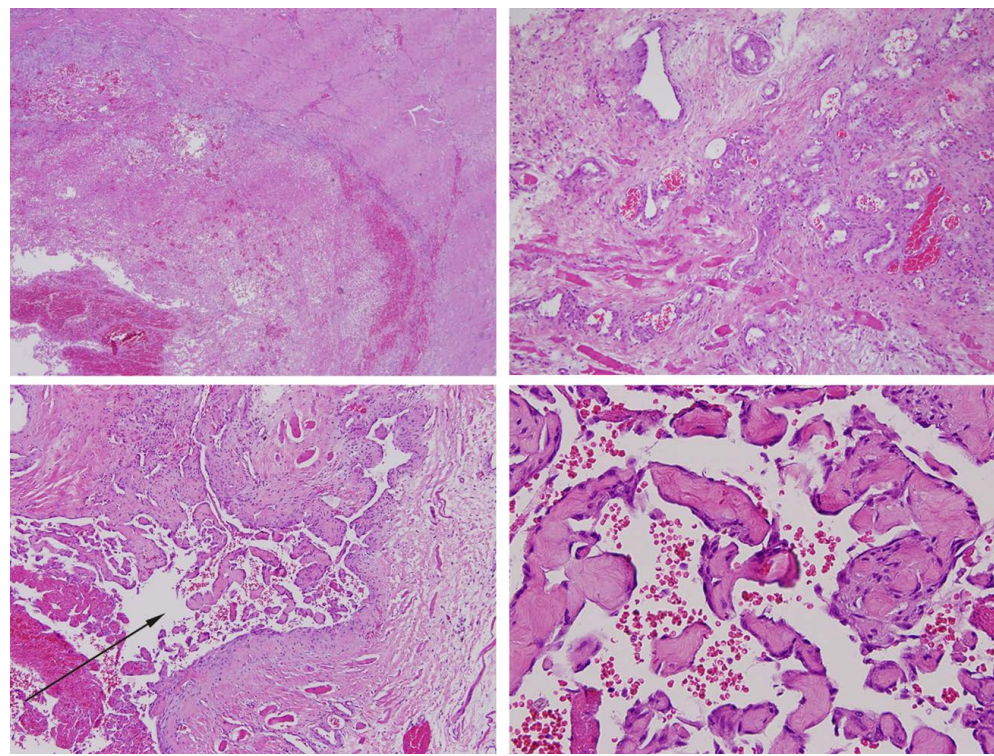

Figure 3. Left top: intramuscular hemangioma with thrombus formation; right top: intramuscular hemangioma; left bottom: intravascular papillary endothelial hyperplasia (arrow) (original magnification $\times 100$ ). Right bottom: papillary endothelial hyperplasia (original magnification $\times 300$ ). 
case. Among these 23 cases [7]-[12], 8 were reported in the renal vein (8/23, 35\%), 3 in the adrenal gland (3/23, $13 \%), 5$ in the gastrointestinal tract $(5 / 23,22 \%), 1$ in the liver (1/23, $4 \%), 4$ in the bladder $(4 / 23,18 \%), 1$ with a multifocal lesion in the retroperitoneum and spine $(1 / 23,4 \%)$, and the current case in the retroperitoneum $(1 / 23$, $4 \%)$. Among the IPEH originating from the gastrointestinal tract, renal vein, and bladder, most were secondary to a comorbidity such as arteriovenous malformation ( 1 in the renal allograft and 2 in the jejunum), paroxysmal nocturnal hemoglobinuria ( 2 in the gastrointestinal tract), radiation therapy (4 in the bladder), and Wegener granulomatosis (1 in the spine and retroperitoneum). However, the IPEH from the adrenal gland and liver mostly had an unknown etiology.

IPEH is usually asymptomatic and often an accidental finding in radiological studies. However, uncommon symptoms resulting from tumor bleeding or peripheral organ compression such as epigastralgia, inguinal pain, low back pain, melena, and hematuria have been reported [7] [8]. No previous published case of a patient presenting with acute sciatica complicated with lumbosacral plexopathy was found in our literature review. This clinical presentation is very rare and mimics lumbosacral radiculopathy, however physicians should be aware of the possibility of retroperitoneal tumors when making a differential diagnosis.

The preoperative differentiation of IPEH is difficult because of its nonspecific clinical symptoms and radiographic features. IPEH may grow outside the vascular lumen after rupture of the vessel and spill over into the surrounding structures, which resembles invasive angiosarcoma. However, the malignant features in angiosarcoma such as high mitotic index, pleomorphism, or extensive necrosis should be absent in IPEH [7] [9]. Thus, surgical treatment with a histological diagnosis is necessary to avoid unnecessary chemotherapy or radiotherapy [7]. Fortunately, the prognosis of IPEH is excellent after surgery, as seen in the current case.

\section{Conclusion}

We present the first case of a huge retroperitoneal intramuscular hemangioma occurring concurrently with IPEH and complicated with lumbosacral plexopathy. This case illustrates a rare condition of coexisting benign tumors leading to progressive neurological deficits.

\section{References}

[1] Godar, M., Yuan, Q., Shakya, R., Xia, Y. and Zhang, P. (2013) Mixed Capillary Venous Retroperitoneal Hemangioma. Case Reports in Radiology, 2013, Article ID: 258352.

[2] Nakatsuka, S., Shigeta, N., Ojima, Y., Kimura, H., Nagano, T. and Ito, K. (2012) A Large Retroperitoneal Cystic Venous Malformation Mimicking Bilateral Ovarian Cystic Tumors. Archives of Gynecology and Obstetrics, 286, 10111014. http://dx.doi.org/10.1007/s00404-012-2395-2

[3] Petry, M., Brown, M.A., Hesselink, J.R. and Imbesi, S.G. (2009) Multifocal Intravascular Papillary Endothelial Hyperplasia in the Retroperitoneum and Spine: A Case Report and Review of the Literature. Journal of Magnetic Resonance Imaging, 29, 957-961. http://dx.doi.org/10.1002/jmri.21724

[4] Masson, P. (1923) Hémangiendothéliome VégétantIntravasculaire. Bulletins de la SociétéAnatomique de Paris, 93, 517-523.

[5] Henschen, F. (1932) L’endovasculite Proliférante Thrombopoïétiquedans la Lésion Vasculaire Locale. Annalesd'anatomiepathologique, 9, 113-121.

[6] Clearkin, K.P. and Enzinger, F.M. (1976) Intravascular Papillary Endothelial Hyperplasia. Archives of Pathology \& Laboratory Medicine, 100, 441-444.

[7] Meadows, M.C., Sun, X., Dardik, M., Tarantino, D.R. and Chamberlain, R.S. (2010) Intraabdominal Intravascular Papillary Endothelial Hyperplasia (Masson’s Tumor): A Rare and Novel Cause of Gastrointestinal Bleeding. Case Reports in Gastroenterology, 4, 124-132.

[8] Jazaerly, T., Jaratli, H., Sakr, W., et al. (2011) Intravascular Papillary Endothelial Hyperplasia of the Bladder: Case Report and Review of the Literature. Avicenna Journal of Medicine, 1, 55-57.

[9] Nassif, A.E., Pozzobon, H.J., Azevedo, E.Z., Taguchi, W.S. and Gomes, R.X. (2009) Papillary Endothelial Hyperplasia of Adrenal: Case Report. Revista do Colegio Brasileiro de Cirurgioes, 36, 277-278. http://dx.doi.org/10.1590/S0100-69912009000300018

[10] Pelosi, G., Sonzogni, A. and Viale, G. (2011) Intravascular Papillary Endothelial Hyperplasia of the Renal Vein. International Journal of Surgical Pathology, 19, 518-520. http://dx.doi.org/10.1177/1066896909341800

[11] Rizza, V., Coletti, G., Di Cocco, P., Mazzotta, C., Famulari, A. and Pisani, F. (2009) Serious Renal Hemorrhage in 
Masson Tumor. Transplantation Proceedings, 41, 1402-1404. http://dx.doi.org/10.1016/j.transproceed.2009.03.010

[12] Tavora, F., Montgomery, E. and Epstein, J.I. (2008) A Series of Vascular Tumors and Tumorlike Lesions of the Bladder. The American Journal of Surgical Pathology, 32, 1213-1219. http://dx.doi.org/10.1097/PAS.0b013e31816293c5 